\title{
INVARIANT SUBSPACES OF INFINITE CODIMENSION FOR SOME NONNORMAL OPERATORS
}

\author{
KEVIN CLANCEY
}

Abstract. Let $\varphi \in C^{\prime}[-1,1]$. For $f \in L^{2}(-1,1)$ define

$$
T_{\varphi} f(s)=s f(s)+\frac{\varphi(s)}{\pi} \int_{-1}^{1 *} \frac{\bar{\varphi} f(t)}{s-t} d t
$$

Our main result says $T_{\varphi}$ has invariant subspaces of infinite codimension.

Introduction. For $\varphi \in C^{\prime}[-1,1]$, consider the singular integral operator defined on $f \in L^{2}(-1,1)$ by

$$
T_{\varphi} f(s)=s f(s)+\frac{\varphi(s)}{\pi} \int_{-1}^{1 *} \frac{\bar{\varphi} f(t)}{s-t} d t, \text { a.e. } s \in[-1,1] .
$$

If the singular integral is interpreted as a Cauchy principal value then $T_{\varphi}$ defines a bounded operator on $L^{2}(-1,1)$. It is known that the operators $T_{\varphi}$ have invariant subspaces arising from eigenvalues of $T_{\varphi}^{*}$. It will be a corollary to our main result (Theorem 1) that $T_{\varphi}$ has invariant subspaces of infinite codimension.

1. An operator $A$ on a Hilbert space $\mathscr{H}$ is said to be hyponormal in case its self-adjoint self-commutator $D=A^{*} A-A A^{*}$ is positive semidefinite $(D \geqq 0)$. An operator $A$ will be called completely nonnormal in case there are no subspaces reducing the operator on which the operator is normal.

The operators $T_{\varphi}$ defined by (1) are hyponormal. Indeed $T_{\varphi}^{*} T_{\varphi}-T_{\varphi} T_{\varphi}^{*}=$ $(2 / \pi)\langle, \varphi\rangle \varphi$. In the cases where $\varphi(t) \neq 0$ a.e. (which we will assume from now on) the operator $T_{\varphi}$ is completely nonnormal.

A point $\lambda \in C$ will be called a bare point of a set $F \subset C$ in case there is a circle $C_{\lambda}$ such that $C_{\lambda} \cap F=\{\lambda\}$ and $\left(C_{\lambda}\right)^{\circ}$ (for $E \subset C, E^{\circ}$ denotes the interior of $E$ ) is contained in the complement of $F$. We will denote the spectrum of an operator $A$ by $\operatorname{sp}(A)$. The number $r_{\mathrm{sp}}(A)=\sup \{|\lambda|: \lambda \in \operatorname{sp}(A)\}$ is called the spectral radius of the operator $A$. An operator $A$ is said to be normaloid

Received by the editors November 29, 1971.

AMS 1969 subject classifications. Primary 4615, 4710.

Key words and phrases. Hyponormal operator, singular integral, invariant subspaces.

(c) American Mathematical Society 1973 
in case $r_{\mathrm{sp}}(A)=\|A\|$. Hyponormal operators are normaloid (Stampfli [9, Theorem 1]).

A sequence $\left\{\lambda_{n}\right\}_{n=1}^{\infty}$ of points in the unit disc is called a Blaschke sequence in case $\sum_{n=1}^{\infty} 1-\left|\lambda_{n}\right|<\infty$. A subset of the unit disc is a zero set for a nonzero bounded analytic function if and only if it is a Blaschke sequence (see, e.g. Rudin [6, p. 302]).

We will also use the following result due to von Neumann (for a proof, see Halmos [3, Problem 180]).

THEOREM $v N$. If $p$ is any polynomial and $A$ an operator of norm at most 1 , then $\|p(A)\| \leqq \max \{|p(\lambda)|:|\lambda| \leqq 1\}$.

The argument used in the following theorem is similar to the argument used in Shields and Wallen [8, Lemma 5].

THEOREM 1. Assume $A$ is completely nonnormal and hyponormal on $\mathscr{H}$. Suppose $\left\{\bar{\lambda}_{n}\right\}_{n=1}^{\infty}$ is a sequence of eigenvalues of $A^{*}$ with eigenvectors $g_{n}$ such that $\lambda_{n} \rightarrow \lambda_{0}$, where $\lambda_{0}$ is a bare point of $\operatorname{sp}(A)$. Then for some infinite subsequence $\operatorname{span}\left(g_{n_{k}}\right)_{k=1}^{\infty} \neq \mathscr{H}^{\prime}$.

Proof. Let $C_{\lambda_{0}}=\left\{\hat{\lambda}:\left|\lambda-\mu_{0}\right|=r\right\}$ be a circle such that $C_{\lambda_{0}} \cap \operatorname{sp}(A)=\left\{\lambda_{0}\right\}$ and $C_{\lambda_{0}}^{\circ} \subset C \backslash \operatorname{sp}(A)$. Consider the operator

$$
A_{1}=\left[\left(A-\mu_{0}\right)^{-1}-\mu_{0} I\right] /\left\|\left(A-\mu_{0}\right)^{-1}-\mu_{0} I\right\| .
$$

Clearly $\left\|A_{1}\right\|=1$ and $\bar{\mu}_{n}=\left[\left(\overline{\hat{\lambda}}_{n}-\bar{\mu}_{0}\right)^{-1}-\bar{\mu}_{0}\right] /\left\|\left(A-\mu_{0}\right)^{-1}-\mu_{0} I\right\|$ is a sequence of eigenvalues of $A_{1}^{*}$. One sees easily that $\left|\mu_{n}\right| \rightarrow r_{\mathrm{sp}}\left(A_{1}\right)=\left\|A_{1}\right\|=1$. The identity $r_{\mathrm{sp}}\left(A_{1}\right)=\left\|A_{1}\right\|$ holds since inverses of hyponormal operators are hyponormal (see Stampfli [10, Lemma 1]). Pick a subsequence $\left\{\mu_{n_{k}}\right\}_{k=1}^{\infty}$ of the $\mu_{n}$ 's such that $\sum_{k=1}^{\infty}\left[1-\left|\mu_{n_{k}}\right|\right]<\infty$. Let $B(z)$ be a bounded analytic function in the unit disc with $B(z)=0$ if and only if $z=\mu_{n_{k}}$ for some $k \geqq 2$. The Fejér means $P_{n}(z)$ of the sequence of $n$th partial sums of the power series expansion of $B(z)$ form a bounded sequence of polynomials converging uniformly on compact subsets of the disc to $B(z)$. Choose a $g \in \mathscr{H}$ such that $\left(g, g_{n_{1}}\right) \neq 0$. Since $\left\|A_{1}\right\| \leqq 1$, by Theorem $v N, P_{k}\left(A_{1}\right) g$ is a bounded sequence in $\mathscr{H}$. We select a weakly convergent subsequence $P_{k_{j}}\left(A_{1}\right) g \rightarrow g^{\prime}$. Then

$$
\left(g^{\prime}, g_{n_{p}}\right)=\operatorname{Lim}_{j}\left(P_{k_{j}}\left(A_{1}\right) g, g_{n_{p}}\right)=\operatorname{Lim}_{j} P_{k_{j}}\left(\mu_{n_{p}}\right)\left(g, g_{n_{p}}\right)=B\left(\mu_{n_{p}}\right)\left(g, g_{n_{p}}\right) \text {. }
$$

It follows that $g^{\prime} \neq 0$ and $g^{\prime} \perp g_{n_{k}}$ for $k \geqq 2$. This completes the proof.

It should be remarked that Theorem 1 is true, for example, if $(a T+b) /(c T+d)$ is normaloid whenever $(a T+b) /(c T+d)$ is bounded.

2. In this section we will describe the spectrum of the operators $T_{\varphi}$ for $\varphi \in C^{\prime}[-1,1]$. It will then be clear that $T_{\varphi}$ has invariant subspaces of 
infinite codimension. Actually the spectrum of $T_{\varphi}$ for $\varphi \in C^{\prime}[-1,1]$ was described by Putnam [4]. We will give a slightly improved description.

A completely hyponormal operator has no eigenvalues. Indeed $A^{*} A \geqq$ $A A^{*}$ says $\left.\|(A-\lambda I) x\|^{2} \geqq \| A^{*}-\bar{\lambda} I\right) x \|^{2}$ for all $\lambda \in C$. The next lemma establishes that eigenvalues of $T_{\varphi}^{*}$ have unit multiplicity.

LEMMA 1. Suppose $A$ is a completely nonnormal operator such that $A^{*} A-A A^{*}=\langle, \varphi\rangle \varphi$. Then $\operatorname{dim} \operatorname{ker} A^{*} \leqq 1$.

Proof. Since $A^{*} A \geqq A A^{*}, \operatorname{ker} A=(0)$. Suppose now $f_{1}, f_{2}$ are two nonzero elements of $\operatorname{ker} A^{*}$. Then $A^{*} A f_{i}=\left\langle f_{i}, \varphi\right\rangle \varphi$. Since ker $A=(0)$ it follows that $\left\langle f_{i}, \varphi\right\rangle \neq 0, i=1,2$. Therefore, $A^{*} A\left[f_{1}-\left(\left\langle f_{1}, \varphi\right\rangle /\left\langle f_{2}, \varphi\right\rangle\right) f_{2}\right]=0$. Again since $\operatorname{ker} A=(0)$ we must have $\left\langle f_{2}, \varphi\right\rangle f_{1}=\left\langle f_{1}, \varphi\right\rangle f_{2}$ and this completes the proof.

Recall that an operator $T$ is Fredholm in case the range of $T$ is closed and both $\operatorname{ker} A$ and $\operatorname{ker} A^{*}$ are finite dimensional. In case $T$ is Fredholm we define the index of $T$ by $i(T)=\operatorname{dim} \operatorname{ker} T-\operatorname{dim} \operatorname{ker} T^{*}$.

The next lemma was pointed out to the author by D. N. Clark.

LEMMA 2. Assume that for $\lambda$ in an open set $\Omega, A-\bar{\lambda} I$ is Fredholm, one-toone and $i(A-\bar{\lambda} I)=-1$. Then the eigenfunctions $f_{\lambda}$ satisfying $\left[A^{*}-\lambda I\right] f_{i}=0$ are analytic in $\lambda$.

Proof. Let $\lambda_{0} \in \Omega$. The hypotheses imply that $A^{*}-\lambda_{0}$ has a right inverse $R_{\lambda_{0}}$ satisfying $\left(A^{*}-\lambda_{0} I\right) R_{\lambda_{0}}=I$. It is easy to see that $f_{\lambda}=\left[A^{*}-\lambda_{0}\right] R_{\lambda} f_{\lambda_{0}}$, for $\lambda \in \Omega$. Analyticity follows since, for $\left|\lambda-\lambda_{0}\right|<\left\|R_{\lambda_{0}}\right\|^{-1}, R_{\lambda}$ has the form $R_{\lambda}=R_{\lambda_{0}} \sum_{n=0}^{\infty}\left(\lambda_{i}-\lambda_{0}\right)^{n} R_{\lambda_{0}}^{n}$.

Let $\mathscr{K}$ denote the ideal of compact operators acting on $\mathscr{H}$. The essential spectrum of an operator $A \in \mathscr{B}(\mathscr{H})$ is the spectrum of the coset determined by $A$ in the Calkin algebra $\mathscr{B}(\mathscr{H}) / \mathscr{K}$. The essential spectrum is the set of complex $\lambda$ such that $A-\lambda$ is not Fredholm (Schwartz [7, Lemma 1]).

Schwartz [7, Theorem 4] has shown that the essential spectrum of $T_{\varphi}$ is the boundary of the curvilinear rectangle $R_{\varphi}=\left\{z=x+i y:-|\varphi(x)|^{2} \leqq y \leqq\right.$ $\left.|\varphi(x)|^{2},-1 \leqq x \leqq 1\right\}$. Now it is known that the spectrum of a nonnormal hyponormal operator $T$ must have positive Lebesgue planar measure. This result is due to Putnam [5] and when $T^{*} T-T T^{*}$ is compact it is due to Clancey [2]. Using Lemmas 1 and 2 and the above remarks we can conclude:

THEOREM 2. The spectrum of $T_{\varphi}$ is $R_{\varphi}$. The boundary of $R_{\varphi}$ is the essential spectrum of $T_{\varphi}$. Each point $\lambda \in R_{\varphi}^{\circ}$ is an eigenvalue of $T_{\varphi}^{*}$ of unit multiplicity. If $f_{\lambda}$ denotes the eigenfunction of $T_{\varphi}^{*}$ corresponding to $\lambda \in R_{\varphi}^{\circ}$ then $f_{\lambda}$ is an $L^{2}$-valued analytic function on $R_{\varphi}^{\circ}$. 
It is now obvious from Theorems 1 and 2 that whenever $\lambda_{n}$ is a sequence in $R_{\varphi}^{\circ}, \lambda_{n} \rightarrow \lambda$ where $\lambda$ is boundary point of $R_{\varphi}$, then some infinite subsequence of the eigenfunctions $f_{n}$ of $T_{\varphi}^{*}$ corresponding to $\lambda_{n}$ fails to span $L^{2}$.

It should be remarked that one can compute the eigenfunctions of $T_{\varphi}^{*}$ explicitly. See, for example, Tricomi [11, Chapter 4, §4]. An interesting problem is to prove or disprove that the set of all eigenfunctions $f_{\lambda}$ of $T_{\varphi}^{*}$ for $\lambda \in R_{\varphi}^{\circ} \operatorname{span} L^{2}(-1,1)$.

In the special case $\varphi(t)=\left(1-t^{2}\right)^{1 / 4}$ the operator $T_{\varphi}$ is the unilateral shift (see Clancey [1]). This is the only operator of the form $T_{\varphi}$ where the complete structure of the invariant subspaces is known.

\section{REFERENCES}

1. K. F. Clancey, On the subnormality of some singular integral operators (preprint).

2. - Seminormal operators with compact self-commutators, Proc. Amer. Math. Soc. 26 (1970), 447-454. MR 42 \#885.

3. P. R. Halmos, A Hilbert space problem book, Van Nostrand, Princeton, N.J., 1967. MR 34 \#8178.

4. C. R. Putnam, The spectra of semi-normal singular integral operators, Canad. J. Math. 22 (1970), 134-150. MR 41 \#4315.

5. —, An inequality for the area of hyponormal spectra, Math. Z. 116 (1970), 323-330. MR 42 \#5085.

6. W. Rudin, Real and complex analysis, McGraw-Hill, New York, 1966. MR 35 \#1420.

7. J. T. Schwartz, Some results on the spectra and spectral resolutions of a class of singular integral operators, Comm. Pure Appl. Math. 15 (1962), 75-90. MR 29 \#480.

8. A. L. Shields and L. J. Wallen, The commutants of certain Hilbert space operators (preprint).

9. J. G. Stampfli, Hyponormal operators, Pacific J. Math. 12 (1962), 1453-1458. MR 26 \#6772.

10. - Hyponormal operators and spectral density, Trans. Amer. Math. Soc. 117 (1965), 469-476. MR 30 \#3375.

11. F. G. Tricomi, Integral equations, Interscience, New York, 1968.

Department of Mathematics, University of Georgia, Athens, Georgia 30601 